\title{
Imaging and chemotherapy cardiotoxicity: A long-playing story still seeking precision and improved outcomes/management data
}

\author{
William A. Van Decker, $M^{a}$ \\ a Temple University School of Medicine, Philadelphia, PA
}

Received Aug 11, 2015; accepted Aug 11, 2015 doi: $10.1007 / \mathrm{s} 12350-015-0274-5$

\section{HISTORICAL PERSPECTIVE}

See related article, pp. 87-97

Doctors have always recognized that every patient is unique, and doctors have always tried to tailor their treatments as best as they can to individuals. ... What if figuring out the right dose of medicine was as simple as taking our temperature?

U.S. President Barack Obama, State of the Union Address, January 30, 2015. Topic Precision Medicine

The ability to define a treatment plan usually entails the clinical judgment of risks and benefits of any treatment option for a given individual patient based on large-scale clinical trials, obtaining patient-physician consensus on the individualized treatment plan, and then initiating a monitoring plan to assess effectiveness and assess side effect profile. When imaging is a portion of the monitoring program for side effect profile, two issues arise. First, what is the reproducible precision of the technique for defining a clinical side effect of importance at the correct clinical stage. Second, what is the utility of the technique for defining a specific directly actionable marker for a defined standardized intervention that is shown to decrease the side effect without dramatically decreasing the treatment efficacy. A clear example of such a clinical conundrum exists in the longrunning tale of Chemotherapy Cardiotoxicity and Cardiology Clinical Care/Cardiac Imaging.

Reprint requests: William A. Van Decker, MD, Temple University School of Medicine, Philadelphia, PA; william.vandecker@tuhs. temple.edu

J Nucl Cardiol 2016;23:98-100.

$1071-3581 / \$ 34.00$

Copyright (C) 2015 American Society of Nuclear Cardiology.
The Oncology Community has made great strides in the treatment of cancer over the past 5 decades, significantly improving survival rates. ${ }^{1}$ This has been especially true in breast cancer, a disease process with unfortunately high prevalence. Yet many chemotherapy drugs 2,3 (most notably, anthracyclines, cyclophosphamide, Her2-neu-targeted agents) have subclinical, or the potential for clinical, cardiac side effects. The most dramatic of the clinical side effects tends to be fall in left ventricular ejection fraction (LVEF) and for some patients full-blown symptomatic congestive heart failure. Fortunately, the overall risk remains lower than the potential benefits of cancer therapy but still argues the need for precise methods to track and targeted interventions at correct times to even further improve clinical outcomes. The mechanism of myocardial cellular dysfunction leading to LVEF pathology from anthracyclines or Cyclophosphamide which tend to be dose related and more irreversible as opposed to the Her2-neu (present in $20-25 \%$ of breast cancer)-targeted agents which seem to tend to be not dose related and more reversible appears quite different adding further complexity to the clinical decision process. This is especially true since both classes of agents are frequently of dramatic benefit in breast oncology, but the combination of anthracycline chemotherapy with Her2-neu agents appears to create additive risk for myocardial dysfunction. ${ }^{4-6}$ As time has gone by, some believe that the risk is not only during the active chemotherapy treatment period of these agents but perhaps can also occur years or decades later (at least with anthracyclines or cyclophosphamide) which has helped fuel the concept of Cardio-Oncology Programs.

The potential role of Cardiac Imaging in monitoring LVEF for adverse effects was established in a landmark manuscript by Alexander et al. in 1979. ${ }^{7}$ This study used first-pass radionuclide angiography to serially follow patients on doxorubicin utilizing a technique that was mathematically reproducible and without geometric assumptions. This paper clearly documented the potential 
development of both asymptomatic and symptomatic fall in LVEF and also clearly documented the dose relationship with LVEF abnormalities usually occurring at doses $>350 \mathrm{mg} / \mathrm{m}^{2}$ of doxorubicin. The manuscript also pointed out the unreliability of systolic time intervals (another marker of LV performance) to predict clinical LV dysfunction. Perhaps more astutely, the manuscript referenced a paper by Bristow et al. ${ }^{8}$ showing endomyocardial biopsy data with doxorubicin with histologic changes below $350 \mathrm{mg} / \mathrm{m}^{2}$ that did not predict clinical fall in LVEF or cardiac symptoms. It went on to allude that subclinical cardiac findings may not be appropriate targets if they limit clinical oncologic effectiveness by stopping meds early. A follow-up study by this group (Choi et al. ${ }^{9}$ ) explored the possibility of monitoring doxorubicin therapy by first-pass radionuclide angiography in patients with starting abnormal LVEFs.

Over the years, many studies of varying imaging modalities to correlate LVEFs between techniques including Multi-Gated Acquisition (MUGA) radio nuclear acquisitions, echocardiography (2D and 3D), Cath ventriculogram, cardiac MR, and cardiac CT have been conducted which can be (and have been) extrapolated to the chemo population. ${ }^{10}$ All have essentially showed "'ballpark' correlation of LVEF determination. Additionally, other potential markers for early LV dysfunction prior to LVEF fall in the chemo population have been explored including echo Doppler diastolic parameters, echo global longitudinal strain, ${ }^{11}$ and the biomarkers of troponin and brain natriuretic peptide (BNP).

Still, to this day, LVEF most commonly by either echo or MUGA has remained the clinical standard for following patients on chemo medications with potential cardiotoxicity. Several Groups in Canada (Mackey et al. ${ }^{12}$ ) and Europe (Bovelli et al. ${ }^{13}$ and Curigliano ${ }^{14}$ On Behalf of ESMO) have generated guidelines based on the most recent Breast Cancer Trials for LVEF monitoring and recommendations for dose adjustments based on LVEF findings.

While the therapy of clinical heart failure follows standard regimens and while there are some data on the early use of ACE inhibitors and beta blockers on those with asymptomatic fall in LVEF (Cardinale et al. ${ }^{15}$ ), current available data focuses most on dose adjustments and "holds" of the chemotherapy medications themselves.

\section{GATED TOMOGRAPHIC RADIONUCLIDE ANGIOGRAPHY WITH CZT DETECTOR GAMMA CAMERA}

In this issue of the Journal, Haarmark et al. report on Pre-Chemotherapy LVEFs and volumes and right ventricular ejection fractions and volumes using (1). Gated
Tomographic (not planar) Radionuclide Angiography (i.e., tomographic MUGA), (2). Technetium-99m-labeled human serum albumin (HSA), not labeled red blood cells, and on a (3). Cadmium-zinc-telluride (CZT) detector gamma camera (not sodium iodide). The patients were all pre-chemotherapy patients, and the purpose was to establish normal values utilizing this technique in this population.

There are good theoretical reasons for obtaining data in this manner. MUGA LVEF is not geometrically based. SPECT format may allow better differentiation of ventricular borders, and calculated LVEF may be "'more precise." Additionally, the advances in spatial resolution/count statistics of CZT may also help create more "precise", LVEF. Most importantly, if a technique will be used to follow key patient parameters serially, then a validation and normative database needs be established for both the equipment and the acquisition parameters together.

The study is well constructed and well reported, but there are some technical concerns that may be raised here. The LVEF standard deviation (SD) is $68.3 \pm 9.2 \%$ in a patient population where a $10 \%$ difference (serial) would be considered significant. The RVEF SD is $47.1 \pm 9.0 \%$ yielding an author-defined lower limit of normal as 2 SD below mean of $29 \%$ (quite a low number to be considered "normal"),

The study at best is a prelude to further work needed for this technical validation. Before we define a "precise" technique, we must define it to be accurate enough to be reproducible. Two serial studies with similar numerical results prior to medications may help in that regard. Most importantly, the data will really take on marked clinical significance only once pre and post chemo serial studies are evaluated side by side and at varying dose and time intervals. Until then, this remains an isolated normative dataset study.

In anticipation, the key hope would be that data acquired in this manner will allow more solid clinical decision making with this patient population and define for us "a reliable specific (or sensitive?) target marker', on which we can clinically act directly to improve clinical outcomes and care.

\section{THE FUTURE}

All well-done medical science studies, even technical radiographic ones, give us the opportunity to think about where we are and what we need to do to improve clinically in the future (and at what "cost", since value $=$ quality divided by cost).

LVEFs/RVEFs/LV and RV volumes can be reported by innumerable technologies and are everyday. We use them as a "ballpark" in the medical manage- 
ment of heart failure and risk stratification in heart failure and coronary heart disease. We wish them to be more "precise" when deciding on Internal Cardio defibrillator Devices when we think 35\% ( \pm what degree of standard deviation?). Yet, the demands of serial LVEF in chemotherapy may sharpen our focus on the LVEF story. What is the most precise reproducible technique with the narrowest SD? Where (and to what degree) does precision add value? Does each technique need its own standardized acquisition or is only the standardization within an institution/practice important? Is there independent information in volumes in assessing ventricular performance and should they always be clinically reported? Should critical LVEFs in nuclear be by first pass or list mode to avoid the rhythm question rather than "frame gating" or does it matter less in the chemo population who most tend to be in sinus rhythm? Does a CZT crystal change LVEF and volumes in planar MUGA acquisitions compared to sodium iodide? Should pivotal clinical trials that use the parameter of LVEF obtain it by several measures and report each individually, use only one and mandate that be the standard for measurement for that clinical question, or obtain it in one manner and allow test substitution based on the clinicians judgement of precision overlay? How does nuclear return to a major role in LV function testing ("prove added quality") when most Radiology Benefit Managers have subjugated it due to "cost"?

More to the current specific clinical question: Is LVEF the best way to follow chemo patients or should we be seeking more "subclinical markers"? (A recent editorial by Yeh et al. ${ }^{16}$ on this topic points out that "A screening test is considered cost efficient only if early detection will lead to intervention that improves outcomes.") and "A limited number of studies have evaluated the benefit of early intervention in asymptomatic patients with subclinical LV dysfunction with contradictory results." ${ }^{15,17}$ ). As the field of CardioOncology evolves, will there be separate recommendations for anthracycline monitoring versus Her2-neu monitoring versus anthracycline sequentially followed by Her2-neu monitoring? Who will determine the consensus on those recommendations given all the stakeholders with different knowledge bases? What is the optimum timing of monitoring and how long should it continue for (on a "cost effective" basis)? Will we return to the search for "cardio protective adjuncts" for these medications rather than primary medication adjustments and stops?

Much work to be done.

\section{Disclosures}

I have no financial disclosures.

\section{References}

1. Siegel R, Miller K, Jemal A. Cancer statistics, 2015. CA Cancer J Clin 2015;65:5-29.

2. Minow R, Benjamin R, Gottlieb J. Adriamycin cardiomyopathy: An overview with determination of risk factors. Cancer Chemother Rep 1975;6:195-201.

3. Perik P, deKorte A, van Veldhulsen D, Gietema JA, Sleijfer DT, De Vries E. Cardiotoxicity associated with the use of Trastuzumab in breast cancer patients. Expert Rev Anticancer Ther 2007;7:1763-71.

4. Piccart-Gebhart M, Proctor M, Leyland-Jones B, Goldhirsch A, Untch M, Smith I, et al. Trastuzumab after adjuvant chemotherapy in HER2 positive breast cancer. NEJM 2005;353:1659-72.

5. Slamon D, Eiermann W, Robert N, Pienkowski T, Martin M, Press $\mathrm{M}$, et al. Adjuvant trastuzumab in HER2 positive breast cancer. NEJM 2011;365:1273-83.

6. Perez E, Ramond E, Suman V, Jeong JH, Davidson NE, Geyer CE, et al. Four year follow up of Trastuzumab plus adjuvant chemotherapy for operable human epidermal growth factor receptor 2 positive breast cáncer: Joint Analysis of data from NCCTG N 9831 and NSABP B-31. J Clin Oncol 2011;20:3366-73.

7. Alexander J, Dainiak N, Berger H, Goldman 1, Johnstone D, Reduto L, et al. Serial assessment of doxorubicin cardiotoxicity with quantitative radionuclide angiocardiography. NEJM 1979;300:278-83.

8. Bristow M, Mason J, Billingham M, Daniels JR. Doxorubicin cardiomyopathy: Evaluation by phonocardiography, endomyocardial biopsy, and cardiac catheterization. Ann Intern Med 1978;88:168-75.

9. Choi B, Berger H, Schwartz P, Alexander J, Gottschalk A, Zaret BL. Serial radionuclide assessment of doxorubicin cardiotoxicity in cancer patients with abnormal baseline resting left ventricular performance. AHJ 1983;106:638-43

10. Plana J, Galdarisi M, Barac A, Ewer MS, Ky B, Scherrer-Crosbie $\mathrm{M}$, et al. Expert consensus for multimodality imaging evaluation of adult patients during and after cancer therapy: A report from the American Society of Echocardiography and the European Association of Cardiovascular Imaging. JASE 2014;27:911-39.

11. Armstrong G, Joshi V, Ness K, Marwick TH, Zhang N, Srivastava $\mathrm{D}$, et al. Comprehensive echocardiographic detection of treatment related cardiac dysfunction in adult survivors of childhood cancer. JACC 2015;65:2511-22.

12. Mackey J, Clemons M, Cote C, Delgado D, Dent S, Patterson A, et al. Cardiac management during adjuvant trastuzumab therapy: Recommendations of the Canadian Trastuzumab Working Group. Curr Oncol 2008;15:24-35.

13. Bovelli D, Plataniotis G, Roila F, ESMO Guidelines Working Group. Cardiotoxicity of chemotherapeutic agents and radiotherapy-related heart disease: ESMO Clinical Practice Guidelines. Ann Oncol 2010;21:V277-82.

14. Curigliano G, Cardinale D, Suter T, Plataniotis E, Azambuja E, Sandri MT, et al. Cardiovascular toxicity induced by chemotherapy, targeted agents and radiotherapy: ESMO Clinical Practice Guidelines. Ann Oncol 2012;23:vii155-66.

15. Cardinale D, Colombo A, Sandri MT, Lamantia G, Colombo N, Civelli M, et al. Prevention of high dose chemotherapy induced cardiotoxicity in high risk patients by angiotensin-converting enzyme inhibition. Circulation 2006;114:2474-81.

16. Yeh E, Vejpongsa P. Subclinical cardiotoxicity associated with cancer therapy. JACC 2015;65:2523-5.

17. Silber JH, Cnaan A, Clark BJ, Paridon SM, Chin AJ, Rychik J, et al. Enalapril to prevent cardiac function decline in long term survivors of pediatric cancer expressed to anthracyclines. J Clin Oncol 2004;22:820-8. 